\title{
Utility and Significance of Gadolinium-Based Contrast Enhancement in Posterior Reversible Encephalopathy Syndrome
}

W e read with considerable interest the article concerning the significance of gadolinium-based contrast enhancement in patients with posterior reversible encephalopathy syndrome (PRES). ${ }^{1}$ The authors have performed a retrospective study on a large patient population and have demonstrated that gadolinium administration is not warranted in these patients because PRES is a clinical-radiologic diagnosis made in appropriate settings and the presence or lack of contrast enhancement does not add to the existing diagnosis algorithm or help in the prognosis of the patients.

A few points merit consideration after reading this article. First, they included 135 patients with a mean age of $40 \pm 20$ years with range from 7 to 82 years. However, they have not separately mentioned the pediatric patients, who have been shown to follow a different clinical-radiologic spectrum. ${ }^{1}$ In our review of pediatric patients with PRES, we have found that contrast enhancement is variably seen in pediatric populations, ranging from $2 \%$ to $53 \%$. This variability is likely due to the multiple etiologies leading to PRES, with imaging in patients having hemato-oncologic malignancies and undergoing chemotherapy predominantly showing enhancement. In our own pediatric PRES cohort, renal disease was seen in $62.5 \%$ of patients and contrast could not be given. Only 2 patients undergoing chemotherapy showed leptomeningeal enhancement. In addition, pediatric patients commonly show atypical MR imaging findings. ${ }^{2}$ These findings indicate that endothelial dysfunction may be the primary mechanism in addition to loss of autoregulation.

We also did not find any correlation among contrast enhancement, MR imaging severity of disease, and patient outcome in our pediatric PRES cohort. A description from the authors of their pediatric cohort, with underlying diseases and pattern of enhancement, may further add to our existing knowledge.

Second, they compared the clinical outcome with the MR imaging severity index as described previously. However, as per our review, the pediatric population frequently shows atypical findings such as frontal lobe, basal ganglia, and brain stem involvement $^{2}$; thus, the conventional MR imaging severity index, based on location, may not be applicable when assessing the prognostic significance in children.

We find the published article useful because it reinforces our own experience of not using contrast unless some alternative pathology is being considered. A pediatric population in which the predominant etiologic cohort is underlying renal disease may not be given contrast, and other patients with PRES may not benefit much from contrast administration as far as diagnosis and clinical outcome are concerned.

\section{REFERENCES}

1. Karia SJ, Rykken JB, McKinney JK, et al. Utility and significance of gadolinium-based contrast enhancement in posterior reversible encephalopathy syndrome. AJNR Am J Neuroradiol 2016;37:415-22 CrossRef Medline

2. Gupta $\mathrm{V}$, Bhatia $\mathrm{V}$, Khandelwal N, et al. Imaging findings in pediatric posterior reversible encephalopathy syndrome (PRES): 5 years of experience from a tertiary care center in India. J Child Neurol 2016 Apr 12. [Epub ahead of print] CrossRef Medline

(D) Vhatia

(D) Vupta

(D) N. Khandelwal

Department of Radio-Diagnosis Postgraduate Institute of Medical Education and Research

Chandigarh, India 